\title{
Systematic analysis and prediction model construction of alternative splicing events in hepatocellular carcinoma: A study on the basis of large-scale spliceseq data from TCGA
}

\author{
Lingpeng Yang ${ }^{1}$, Yang He ${ }^{1}$, Zifei Zhang ${ }^{1}$, Wentao Wang ${ }^{\text {Corresp. } 1}$ \\ ${ }^{1}$ Department of Liver Surgery \& Liver Transplantation Center, West China Hospital of Sichuan University, Chengdu, Sichuan, China \\ Corresponding Author: Wentao Wang \\ Email address: wwt0510@163.com
}

Growing evidence showed that alternative splicing (AS) event is significantly related to tumor occurrence and progress. This study was performed to make a systematic analysis of AS events and constructed a robust prediction model of hepatocellular carcinoma (HCC). The clinical information and the genes expression profile data of 335 HCC patients were collected from The Cancer Genome Atlas (TCGA). Information of seven types AS events were collected from the TCGA SpliceSeq database. Overall survival (OS) related AS events and splicing factors (SFs) were identified using univariate Cox regression analysis. The corresponding genes of OS-related AS events were sent for gene network analysis and functional enrichment analysis. Optimal OS-related AS events were selected by LASSO regression to construct prediction model using multivariate Cox regression analysis. Prognostic value of the prediction models were assessed by receiver operating characteristic (ROC) curve and Kaplan-Meir survival analysis. The relationship between the Percent Spliced In (PSI) value of OS-related AS events and SFs expression were analyzed using Spearman correlation analysis. And the regulation network was generated by Cytoscape. A total of 34163 AS events were identified, which consist of 3482 OS-related AS events. UBB, UBE2D3, SF3A1 were the hub genes in the gene network of the top 800 OSrelated AS events. The area under the curve (AUC) of the final prediction model based on seven types OS-related AS events was $0.878,0.843,0.821$ in $1,3,5$ years, respectively. Upon multivariate analysis, risk score (All) served as the risk factor to independently predict OS for HCC patients. SFs HNRNPH3 and HNRNPL were overexpressed in tumor samples and were signifcantly associated with the OS of HCC patients. The regulation network showed prominent correlation between the expression of SFs and OS-related AS events in HCC patients. The final prediction model performs well in predicting the prognosis of HCC patients. And the findings in this study improve our understanding of the association between AS events and HCC. 
1 Systematic analysis and prediction model construction of alternative splicing events in

2 hepatocellular carcinoma: A study on the basis of large-scale spliceseq data from TCGA

3 Lingpeng Yang ${ }^{1}$, Yang $\mathrm{He}^{1}$, Zifei Zhang ${ }^{1}$, Wentao Wang ${ }^{1 *}$

$4{ }^{1}$ Department of Liver Surgery \& Liver Transplantation Center, West China Hospital of Sichuan

5 University, Chengdu, Sichuan, China

6 *Correspondence Wentao Wang, Department of Liver Surgery \& Liver Transplantation Center,

7 West China Hospital of Sichuan University, Chengdu, Sichuan, China.

8 E-mail: wwt0510@163.com

\section{Abstract}

Growing evidence showed that alternative splicing (AS) event is significantly related to tumor occurrence and progress. This study was performed to make a systematic analysis of AS events and constructed a robust prediction model of hepatocellular carcinoma (HCC). The clinical information and the genes expression profile data of $335 \mathrm{HCC}$ patients were collected from The Cancer Genome Atlas (TCGA). Information of seven types AS events were collected from the TCGA SpliceSeq database. Overall survival (OS) related AS events and splicing factors (SFs) were identified using univariate Cox regression analysis. The corresponding genes of OSrelated AS events were sent for gene network analysis and functional enrichment analysis. Optimal OS-related AS events were selected by LASSO regression to construct prediction model using multivariate Cox regression analysis. Prognostic value of the prediction models were assessed by receiver operating characteristic (ROC) curve and Kaplan-Meir survival analysis. The relationship between the Percent Spliced In (PSI) value of OS-related AS events and SFs expression were analyzed using Spearman correlation analysis. And the regulation network was generated by Cytoscape. A total of 34163 AS events were identified, which consist of 3482 OSrelated AS events. UBB, UBE2D3, SF3A1 were the hub genes in the gene network of the top 800 OS-related AS events. The area under the curve (AUC) of the final prediction model based on seven types OS-related AS events was $0.878,0.843,0.821$ in $1,3,5$ years, respectively. Upon multivariate analysis, risk score (All) served as the risk factor to independently predict OS for 
HCC patients. SFs HNRNPH3 and HNRNPL were overexpressed in tumor samples and were significantly associated with the OS of HCC patients. The regulation network showed prominent correlation between the expression of SFs and OS-related AS events in HCC patients. The final prediction model performs well in predicting the prognosis of HCC patients. And the findings in this study improve our understanding of the association between AS events and HCC.

\section{Keywords}

Alternative splicing; Hepatocellular carcinoma; Prediction model; Splicing factor; Risk score; Prognosis; Overall survival; TCGA; PSI value; LASSO regression

\section{Introduction}

As the main component of tissues and cells, proteins play a vital role in many life activities. The diversity of proteins contributes to the functional diversity. Alternative splicing (AS) is a significant and ubiquitous post-transcriptional regulatory mechanism that enables eukaryotic cells to generate vast protein diversity based on a limited number of genes (Baralle \& Giudice, 2017). Genome-wide research indicated that up to $95 \%$ of human genes experience some level of AS in physiological processes (Pan et al., 2008; Wang et al., 2008). Precursor mRNA can be transformed to mature mRNA and further produce versatile protein by removing introns and selectively including or excluding specific exons in human multi-exon genes (Kelemen et al., 2013). Recently, growing evidence showed that AS is significantly related to tumor occurrence, progress and therapeutic resistance, and AS is involved in the process of invasion and metastasis of cancer cells (Wan et al., 2019; Oltean \& Bates, 2014). Splicing factor (SF) is the executor of AS events and aberrant expression of SF was associated with oncogenesis process (Dvinge et al., 2016).

Liver cancer ranks the $6^{\text {th }}$ place in terms of global tumor incidence, and it is the $4^{\text {th }}$ leading cause of cancer-related death (Villanueva, 2019). Hepatocellular carcinoma (HCC), one of the frequently seen primary liver tumors, occupies about $80 \%$ of liver cancers. Worldwide, the highest liver cancer morbidity is reported in Asia and Africa. About 75\% liver cancers take place in Asian areas, among which, China accounts for more than half of the total global cases 
55

(McGlynn et al., 2015). Although great progresses have been achieved in diagnosing and treating liver tumor over past few decades, prognosis for liver tumor remains very poor. Liver cancer has become the second most fatal tumor after pancreatic cancer with a 5-year survival rate of $18 \%$ (Jemal et al., 2017). In the recent years, some studies have reported the crucial significance of AS in HCC occurrence and development (Yuan et al., 2017; Luo et al., 2017). However, systematic analysis of the predictive value of AS events in $\mathrm{HCC}$ is scarce.

In this study, we collected RNA-seq data, AS events data and corresponding clinical information of $335 \mathrm{HCC}$ patients from TCGA database. Overall survival (OS) related AS events and splicing factors (SFs) were identified. Additionally, a reliable prediction model on the basis of AS events, correlation network between AS events and SFs were constructed.

\section{Materials and Methods}

\section{TCGA-based data collection}

The genes expression profile data as well as the clinical information of HCC patients had been collected from TCGA (https://cancergenome.nih.gov/). Information of seven types AS events were collected from the TCGA SpliceSeq database (https://bioinformatics.mdanderson.org/TCGASpliceSeq/), including alternate acceptor site (AA), alternate promoter (AP), alternate donor site (AD), alternate terminator (AT), exon skip (ES), mutually exclusive exons (ME), and retained intron (RI). Due to defining perioperative mortality as death that occurs within 30 days of surgery may underestimate 'true' mortality among patients undergoing hepatic resection (Mayo et al., 2011). We only included patients with a survival time more than 90 days, finally, $335 \mathrm{HCC}$ patients were selected in this study. The Percent Spliced In (PSI) value, rating from 0 to 1 which is used to quantify AS events in general. To generate a reliable set of AS events, we applied a series of stringent filters (Percentage of samples with PSI value $\geq 75 \%$, standard deviation of PSI value $>0.1$ and average PSI value $>0.05)$.

8 Every AS event was assigned a unique identifier by combining the gene symbol, the ID number in the SpliceSeq database and splicing type. For instance, in the identifier term "CSAD- 
82

21952-ES", the gene symbol is CSAD, ID number is 21952 and splicing type is ES.

\section{Identification of overall survival related AS events, functional enrichment analysis and} gene network construction

We identified overall survival (OS) related AS events using univariate Cox regression analysis with a $\mathrm{P}$ value $<0.05$. Upset plot which is similar to a Venn diagram, was introduced to depict the intersections between the seven types of AS events in HCC (Khan \& Mathelier, 2017). The corresponding genes of OS-related AS events were sent for functional enrichment analysis. Gene Ontology (GO) and Kyoto Encyclopedia of Genes and Genomes (KEGG) pathways with P value $<0.05$ and false discovery rate $($ FDR $)<0.05$ were considered significantly. The top significant pathways in KEGG and GO were showed with bar plots. Gene network analysis was performed by inputting the corresponding genes of 800 most significant OS-related AS events to Cytoscape and Reactome FI (version 3.7.1), further, hub genes were selected at the same time.

\section{Construction of the prediction model based on AS events}

Least absolute shrinkage and selection operator (LASSO) regression is suitable for the reduction of high-dimensional data to avoid model overfitting (Sauerbrei et al., 2007). When we performed LASSO regression, some variables were eliminated through penalty rules and potential predictors with non-zero coefficients were finally leaved (Gao et al., 2010). We determined the penalty parameter lambda by the cross-validation using the glmnet package. The optimal lambda value corresponding to the minimum value of the cross-validation error mean was identified to determine the potential OS-related AS events (Tibshirani, 1997). We selected the optimal OS-related AS events in each type respectively with nonzero coefficients in the LASSO regression, and constructed the prediction models by using multivariate Cox regression analysis. The final prediction model (all types) was constructed by combing seven types of AS events also selected via LASSO regression.

The prediction model was established according to risk score, and the risk score was calculated by the PSI value of each AS events and the corresponding regression coefficient (lnHR) generated from multivariate Cox regression analysis. The formula is as follows: 
109 Risk score $=\sum_{i}^{n}$ PSI $i * \beta i$, where $\beta$ is the regression coefficient.

110

111

112

113

114

115

116

117

118

119

120

121

122

123

124

125

126

127

128

129

130

131

132

133

134

135

\section{Prognostic value evaluation of the prediction model}

The HCC patients were divided into low and high risk groups according to the median value of the risk score and Kaplan-Meier survival analysis was performed to compare the OS rate between the two groups within five years. The p-values were computed using log-rank test. Furthermore, ROC curves of 1, 3, 5 years were generated to compare the predictive accuracy of each prediction model.

\section{Construction of the regulation network between SFs and AS events}

SF data was obtained from the SpliceAid2 database (http://www.introni.it/splicing.html). The association of SF expression with OS was analyzed using univariate Cox regression. The relationship between the PSI value of OS-related AS events and SF expression were analyzed using Spearman correlation analysis. Regulation network was generated by Cytoscape and Reactome FI (version 3.7.1).

\section{Statistical analysis}

The R software 3.5.0 was utilized for all statistical analysis. A P value $<0.05$ was deemed to be of statistical significance.

\section{Results}

\section{Overview of AS events in HCC cohort}

A flowchart of our study design is showed in Figure 1. We detected a total of 34163 AS events in 8985 genes in 335 HCC patients, comprised of 12327 ES events in 5343 genes, 8087 AT events in 3532 genes, 6352 AP events in 2566 genes, 2666 AA events in 1937 genes, 2331 AD events in 1663 genes, 2263 RI events in 1561 genes and $137 \mathrm{ME}$ events in 135 genes (Table 1). We can find that one single gene undergoes up to six types of AS events from the UpSet plot (Fig 2A). In addition, ES was the most common among seven types of AS events.

\section{The OS-related AS events of HCC}

A total of 3482 OS-related AS events in 2203 genes were detected using univariate Cox analysis, including 1264 ES events in 988 genes, 887 AT events in 534 genes, 643 AP events in 
136

137

138

139

140

141

142

143

144

145

146

147

148

149

150

151

152

153

154

155

156

157

158

159

160

161

162

411 genes, 217 AA events in 203 genes, 231 AD events in 205 genes, 224 RI events in 196 genes and $16 \mathrm{ME}$ events in 16 genes (Table 1). A volcano plot of these AS events was provided in Fig 2B. With the display of UpSet plot, one single gene could have up to four types OS-related AS events (Fig 2C). The top 20 significant OS-related AS events (if available) for each AS type were showed by bubble plots in Fig 3A-3G. Obviously, most of AS events in ES were favorable prognostic elements. However, most of these AS events in RI, AA and AD were adverse prognostic elements.

We established a gene interaction network by sending the corresponding genes of the top 800 most significant OS-related AS events to Cytoscape. The results indicated that UBB, UBE2D3, SF3A1 were the hub genes of this gene network (Fig 4A). Furthermore, 2203 genes from 3482 OS-related AS events were used for KEGG and GO enrichment analysis to explore the pathways and biological functions of the OS-related AS genes. The top 10 significant terms of GO enrichment analysis were presented in Fig 4B. Such as spliceosomal complex, adherens junction in cellular component (CC); damaged DNA binding, cell adhesion molecule binding in molecular function (MF); protein targeting, actin cytoskeleton reorganization in biological process (BP). A total of nine KEGG pathways were identified, such as base excision repair, pyruvate metabolism and PPAR signaling pathway (Fig 4C).

\section{Construction of the prediction model for HCC patients}

We used LASSO regression to select the top 10 optimal OS-related AS events (if available) and then construct the prediction model (Fig 5). Three OS-related AS events were selected for ES; four OS-related AS events for ME; five OS-related AS events for AP and AT; six OS-related AS events for AA, AD and RI; nine OS-related AS events for the final prediction model (Table 2). Risk scores were computed according to the selected AS events, and HCC patients were divided into low and high risk groups on the basis of the median value of risk scores. The distribution of survival status in low and high risk groups, risk score curves and the PSI value heat map of the AS events for eight prediction models were visualized in Fig 6. The results of

Kaplan-Meier survival analysis showed that all of the eight prediction models possess significant 
163

164

165

166

167

168

169

170

171

172

173

174

175

176

177

178

179

180

181

182

183

184

185

186

187

188

189

ability to predict the prognosis of HCC patients between low and high risk group (Fig 7).

However, according the results of ROC curves, the final prediction model exhibited the most powerful predictive efficiency than other models established by one single AS type with the maximum AUCs of $0.878,0.843,0.821$ in $1,3,5$ years ROC curves (Fig 8).

Moreover, we assessed the prognostic value of risk score and other clinicopathological characteristics using univariate and multivariate Cox regression analysis. Univariate analysis suggested that advanced clinical stage $(\mathrm{HR}=2.053,95 \% \mathrm{CI}: 1.553-2.714, \mathrm{p}<0.001)$, high $\mathrm{T}$ classification $(\mathrm{HR}=1.962,95 \% \mathrm{CI}: 1.515-2.542, \mathrm{p}<0.001)$ and high risk score $(\mathrm{All})(\mathrm{HR}=1.105$, 95\% CI: 1.076-1.135, $\mathrm{p}<0.001)$ were associated with poor prognosis (Fig 9A). Upon multivariate analysis, risk score (All) $(\mathrm{HR}=1.101,95 \% \mathrm{CI}: 1.069-1.135, \mathrm{p}<0.001)$ served as the risk factor to independently predict OS for HCC patients (Fig 9B).

\section{The regulation network of SFs and OS-related AS events}

SF is the executor of AS events and aberrant expression of SF was related to oncogenesis process. A total of 71 SFs data were collected from the SpliceAid2 database (http://www.introni.it/splicing.html). We identified $21 \mathrm{SFs}$ associated with OS of HCC patients using univariate analysis. Then Spearman correlation analysis was performed to determine the correlation between the PSI value of OS-related AS events and SF expression. The significant correlations $(|\mathrm{R}| \geq 0.6, \mathrm{p}<0.001)$ were selected to construct the regulation network (Fig $10 \mathrm{~A})$. The regulation network consists of 29 OS-related AS events, of which 16 were adverse AS events (red dots) and 13 were favorable AS events (green dots), were significantly correlated with the 13 SFs (blue dots). We can find that all of the SFs were correlated with multiple AS events and played opposite roles in regulating different AS events. Similarly, a part of the AS events could be regulated by different SFs. This phenomenon partly explains that the same transcript can produce multiple different splicing events. In addition, we can detect that the adverse AS events were positively correlated with SF expression (red lines), whereas the favorable AS events were negatively correlated with SF expression (green lines).

The top four most significant correlations between SFs and OS-related AS events $(|\mathrm{R}| \geq 0.7)$ 
are shown in Fig 10D, 10E, 10H, 10I. The top two counterpart SFs were HNRNPL and HNRNPH3, and the expression of HNRNPL and HNRNPH3 in tumor sample were significantly higher than adjacent normal sample (Fig 10B, 10F). HCC patients were classified into low and high risk groups according to the median value of the two SFs expression. And Kaplan-Meier survival analysis showed statistical difference in survival time between the two groups (Fig 10C, 10G).

\section{Discussion}

AS is a significant regulatory process for generating protein isoforms with a variety of functional characteristics. Abnormality of AS events are closely associated with tumor occurrence, development and metastasis (Oltean \& Bates, 2014; Liu \& Cheng, 2013; Spaethling et al., 2016). In recent decades, with the rapid development of high-throughput sequencing technology, the potential significance of AS events in malignant tumor has achieved great advancement. However, there are few studies focus on the systematic analysis of AS events in HCC patients.

Then we screened OS-related AS events and OS-related SFs in HCC patients via the analysis of TCGA and SpliceAid2 database. A total of 3482 OS-related AS events in 2203 genes and 21 OS-related SFs were detected using univariate Cox analysis, which shows that AS events are ubiquitous in HCC patients and limited SFs can regulate massive AS. The gene interaction network was established using Cytoscape based on the top 800 OS-related AS events, and UBB, UBE2D3, SF3A1 were identified as the hub genes of this gene network. Ubiquitin is a small and highly conserved protein expressed in all eukaryotic cells. Over expression of ubiquitin B (UBB) was reported in non-small cell lung cancer and cervical cancer, UBB may serve as a potential therapy and prevention target (Tang et al., 2015; Tian et al., 2013). Nevertheless, the relationship between HCC and UBB is not clear. Ubiquitin-conjugating enzyme E2D3 (UBE2D3) is a member of the E2 family, which is involved in the ubiquitin proteasome pathway to regulate the basic activities of cells, such as DNA damage response, cell cycle control, apoptosis, and tumorgenesis. A previous study demonstrated that UBE2D3 plays a significant role in the 
217 development of esophageal cancer (Guan et al., 2015). SF3A1 is a critical spliceosome gene

218 which participated in normal splicing events and spliceosome assembly (Chen et al., 2015a).

219 SF3A1 has been reported to be related to susceptibility of breast cancer and lung cancer (Hu et

220 al., 2011; Michailidou et al., 2013). And the mutation of SF3A1 were involved in some cancers,

221 including esophageal adenocarcinoma, osteosarcomas, ovarian carcinoma and gastric cancer

222 (Chen et al., 2015b). Additionally, KEGG and GO enrichment analysis were performed and we

223 found that these genes were closely associated with spliceosomal complex, cell adhesion

224

225

226

227

228

229

230

231

232

233

234

235

236

237

238

239

240

241

242

243 molecule binding, actin cytoskeleton reorganization, base excision repair, etc. From the above mentioned, UBB, UBE2D3 and SF3A1 as the hub genes in this gene interaction network and may be potential targets for the prevention and treatment of $\mathrm{HCC}$ in the future.

In the recent years, with the fast development of high-throughput sequencing, massive potential prognosis biomarkers or therapy targets of tumor were emerging, such as mRNA, miRNA, IncRNA, and methylation (Zhao et al., 2018; Ji et al., 2018; Cai et al., 2019; Wu et al., 2017). However, the focus of these studies is limited to the transcriptome-level analysis. The prognostic value of AS events has a great potential for development. Tremblay et al (Tremblay et al., 2016) firstly investigated differential AS events between HCC sample and normal liver sample based on TCGA database, and they provided an overview of misregulated AS events in different types of HCC (e.g., HBV-related HCC, HCV-related HCC, HBV\&HCV-associated HCC and virus-free HCC). But they did not explore the association between the AS events and the prognosis of HCC patients. Subsequently, some researchers identified OS-related AS events to establish a prediction model in HCC patients (Chen et al., 2019; Zhu et al., 2019). However, Chen et al. reported that the AUC of ROC curve for the final prediction model constructed with 10 AS events was only 0.752 . Zhu et al. constructed the final prediction model with up to 33 AS events, but the AUC of ROC curve was only 0.806. In this study, the final prediction model exhibited the most powerful predictive efficiency than other models established by one single AS type with the maximum AUCs of $0.878,0.843,0.821$ in $1,3,5$ years ROC curves. And our final prediction model was constructed with only nine OS-related AS events. 
244

245

246

247

248

249

250

251

252

253

254

255

256

257

258

259

260

261

262

263

264

265

266

267

268

269

270

SF plays a significant role in the regulation of AS events, which affects the selection of exons and splicing sites by identifying and combining to cis-regulatory elements of pre-mRNA. Aberrant expression of SF was related to oncogenesis process. The regulation network was constructed to show the correlation between the PSI value of OS-related AS events and SF expression. The top two SFs were HNRNPL and HNRNPH3 identified according to the correlation coefficient $(|\mathrm{R}| \geq 0.7)$. Compared to adjacent normal sample, the expression of HNRNPL and HNRNPH3 were higher in tumor sample. And patients with higher expression of HNRNPL and HNRNPH3 had more dismal prognosis. From the regulation network, we found that the adverse AS events were positively correlated with the expression of HNRNPL and HNRNPH3, whereas the favorable AS events were negatively correlated with the expression of HNRNPL and HNRNPH3, which was consistent with the results of survival analysis. HNRNPL and HNRNPH3 are members of HNRNPs family. HNRNPs directly modulate the AS of a group of RNAs and serve as multifunctional RNA-binding proteins for mRNA stabilization, transportation and translation (Fei et al., 2017). Aberrant expression of HNRNPL and its RNA target are closely associated with the proliferation, invasion and metastasis of tumor cells (Kedzierska \& Piekielko-Witkowska, 2017; Geuens et al., 2016). A recent study have demonstrated that HNRNPL was highly expressed in HCC samples and down-regulation of HNRNPL expression can significantly inhibit the proliferation and migration of liver cancer cells (Yau et al., 2013), which was in accordance with our results. However, to the best knowledge of us, there is no research report the actual regulatory mechanism between the two prognostic SFs and OS-related AS events, and further elucidation with in vivo or vitro experiments is urgently needed.

Although the findings in the present study improves our understanding of the association between AS events and HCC, some limitations existed in this study. First, this study was conducted based on the data obtained from one public database with a relatively small sample size. Second, due to the lack of external data, we did not make a cohort verification for the prediction model. Third, we did not perform a functional experiment and could not clearly 
271

272

273

elucidate the underlying mechanism between AS events and SFs in HCC patients. It is essential to carry out functional experiments and clinical trials with a large sample size of HCC patients to confirm the findings of this study in the future.

\section{Conclusions}

In summary, we performed a systematic analysis of AS events in $\mathrm{HCC}$ and constructed prediction models based on OS-related AS events with well performance in predicting the prognosis of HCC patients. The AS events used for the construction of the final prediction model may be the most significant AS events in exploring the latent mechanism in initiation and development of $\mathrm{HCC}$, have a great potential for clinical application as therapeutic and preventive targets of HCC patients. Furthermore, we established a regulation network between SFs and OSrelated AS events. Although the findings in the present study improve our understanding of the association between $\mathrm{AS}$ events and $\mathrm{HCC}$ to some extent. In vitro/vivo function experiments are also urgently needed in the future to understand the mechanism between AS events and SFs in the HCC patients.

\section{Funding}

The current work was supported by the National Natural Science Foundation of China (No. 81770566), the New Medical Technology Foundation of West China Hospital of Sichuan University (No. XJS2016004), and the Science and Technology Program of Sichuan Science and Technology Department (No. 2019YFS0029).

\section{Acknowledgments}

None.

\section{Conflict of interest statement}

The authors declare that there are no conflicts of interest.

\section{References}

Baralle, F.E., and Giudice, J. 2017. Alternative splicing as a regulator of development and tissue identity. Nat Rev Mol Cell Biol 18:437-451. 10.1038/nrm.2017.27

Cai, J., Tong, Y., Huang, L., Xia, L., Guo, H., Wu, H., Kong, X., and Xia, Q. 2019. Identification and validation of a potent multi-mRNA signature for the prediction of early relapse in hepatocellular carcinoma. CARCINOGENESIS 40:840-852. 10.1093/carcin/bgz018

Chen, Q.F., Li, W., Wu, P., Shen, L., and Huang, Z.L. 2019. Alternative splicing events are prognostic in 
hepatocellular carcinoma. Aging (Albany NY) 11:4720-4735. 10.18632/aging.102085

Chen, X., Du H, Liu, B., Zou, L., Chen, W., Yang, Y., Zhu, Y., Gong, Y., Tian, J., Li, F., and Zhong, S. 2015a. The Associations between RNA Splicing Complex Gene SF3A1 Polymorphisms and Colorectal Cancer Risk in a Chinese Population. PLoS One 10:e130377. 10.1371/journal.pone.0130377

Chen, X., Du H, Liu, B., Zou, L., Chen, W., Yang, Y., Zhu, Y., Gong, Y., Tian, J., Li, F., and Zhong, S. 2015b. The Associations between RNA Splicing Complex Gene SF3A1 Polymorphisms and Colorectal Cancer Risk in a Chinese Population. PLoS One 10:e130377. 10.1371/journal.pone.0130377

Dvinge, H., Kim, E., Abdel-Wahab, O., and Bradley, R.K. 2016. RNA splicing factors as oncoproteins and tumour suppressors. NATURE REVIEWS CANCER 16:413-430. 10.1038/nrc.2016.51

Fei, T., Chen, Y., Xiao, T., Li, W., Cato, L., Zhang, P., Cotter, M.B., Bowden, M., Lis, R.T., Zhao, S.G., Wu, Q., Feng, F.Y., Loda, M., He, H.H., Liu, X.S., and Brown, M. 2017. Genome-wide CRISPR screen identifies HNRNPL as a prostate cancer dependency regulating RNA splicing. Proc Natl Acad Sci U S A 114:E5207-E5215. 10.1073/pnas. 1617467114

Gao, J., Kwan, P.W., and Shi, D. 2010. Sparse kernel learning with LASSO and Bayesian inference algorithm. Neural Netw 23:257-264. 10.1016/j.neunet.2009.07.001

Geuens, T., Bouhy, D., and Timmerman, V. 2016. The hnRNP family: insights into their role in health and disease. HUMAN GENETICS 135:851-867. 10.1007/s00439-016-1683-5

Guan, G.G., Wang, W.B., Lei, B.X., Wang, Q.L., Wu, L., Fu, Z.M., Zhou, F.X., and Zhou, Y.F. 2015. UBE2D3 is a positive prognostic factor and is negatively correlated with hTERT expression in esophageal cancer. Oncology Letters 9:1567-1574. 10.3892/ol.2015.2926

Hu, Z., Wu, C., Shi, Y., Guo, H., Zhao, X., Yin, Z., Yang, L., Dai, J., Hu, L., Tan, W., Li, Z., Deng, Q., Wang, J., Wu, W., Jin, G., Jiang, Y., Yu, D., Zhou, G., Chen, H., Guan, P., Chen, Y., Shu, Y., Xu, L., Liu, X., Liu, L., Xu, P., Han, B., Bai, C., Zhao, Y., Zhang, H., Yan, Y., Ma, H., Chen, J., Chu, M., Lu, F., Zhang, Z., Chen, F., Wang, X., Jin, L., Lu, J., Zhou, B., Lu, D., Wu, T., Lin, D., and Shen, H. 2011. A genome-wide association study identifies two new lung cancer susceptibility loci at 13q12.12 and 22q12.2 in Han Chinese. NATURE GENETICS 43:792-796. 10.1038/ng.875

Jemal, A., Ward, E.M., Johnson, C.J., Cronin, K.A., Ma, J., Ryerson, B., Mariotto, A., Lake, A.J., Wilson, R., Sherman, R.L., Anderson, R.N., Henley, S.J., Kohler, B.A., Penberthy, L., Feuer, E.J., and Weir, H.K. 2017. Annual Report to the Nation on the Status of Cancer, 1975-2014, Featuring Survival. J Natl Cancer Inst 109. 10.1093/jnci/djx030

Ji, J., Chen, H., Liu, X.P., Wang, Y.H., Luo, C.L., Zhang, W.W., Xie, W., and Wang, F.B. 2018. A miRNA Combination as Promising Biomarker for Hepatocellular Carcinoma Diagnosis: A Study Based on Bioinformatics Analysis. Journal of Cancer 9:3435-3446. 10.7150/jca.26101

Kedzierska, H., and Piekielko-Witkowska, A. 2017. Splicing factors of SR and hnRNP families as regulators of apoptosis in cancer. CANCER LETTERS 396:53-65. 10.1016/j.canlet.2017.03.013

Kelemen, O., Convertini, P., Zhang, Z., Wen, Y., Shen, M., Falaleeva, M., and Stamm, S. 2013. Function of alternative splicing. GENE 514:1-30. 10.1016/j.gene.2012.07.083

Khan, A., and Mathelier, A. 2017. Intervene: a tool for intersection and visualization of multiple gene or genomic region sets. BMC BIOINFORMATICS 18:287. 10.1186/s12859-017-1708-7

Liu, S., and Cheng, C. 2013. Alternative RNA splicing and cancer. Wiley Interdiscip Rev RNA 4:547-566. 10.1002/wrna. 1178 
Luo, C., Cheng, Y., Liu, Y., Chen, L., Liu, L., Wei, N., Xie, Z., Wu, W., and Feng, Y. 2017. SRSF2 Regulates Alternative Splicing to Drive Hepatocellular Carcinoma Development. CANCER RESEARCH 77:1168-1178. 10.1158/0008-5472.CAN-16-1919

Mayo, S.C., Shore, A.D., Nathan, H., Edil, B.H., Hirose, K., Anders, R.A., Wolfgang, C.L., Schulick, R.D., Choti, M.A., and Pawlik, T.M. 2011. Refining the definition of perioperative mortality following hepatectomy using death within 90 days as the standard criterion. HPB (Oxford) 13:473-482. 10.1111/j.1477-2574.2011.00326.x

McGlynn, K.A., Petrick, J.L., and London, W.T. 2015. Global epidemiology of hepatocellular carcinoma: an emphasis on demographic and regional variability. Clinics in Liver Disease 19:223-238. 10.1016/j.cld.2015.01.001

Michailidou, K., Hall, P., Gonzalez-Neira, A., Ghoussaini, M., Dennis, J., Milne, R.L., Schmidt, M.K., Chang-Claude, J., Bojesen, S.E., Bolla, M.K., Wang, Q., Dicks, E., Lee, A., Turnbull, C., Rahman, N., Fletcher, O., Peto, J., Gibson, L., Dos, S.S.I., Nevanlinna, H., Muranen, T.A., Aittomaki, K., Blomqvist, C., Czene, K., Irwanto, A., Liu, J., Waisfisz, Q., Meijers-Heijboer, H., Adank, M., van der Luijt, R.B., Hein, R., Dahmen, N., Beckman, L., Meindl, A., Schmutzler, R.K., Muller-Myhsok, B., Lichtner, P., Hopper, J.L., Southey, M.C., Makalic, E., Schmidt, D.F., Uitterlinden, A.G., Hofman, A., Hunter, D.J., Chanock, S.J., Vincent, D., Bacot, F., Tessier, D.C., Canisius, S., Wessels, L.F., Haiman, C.A., Shah, M., Luben, R., Brown, J., Luccarini, C., Schoof, N., Humphreys, K., Li, J., Nordestgaard, B.G., Nielsen, S.F., Flyger, H., Couch, F.J., Wang, X., Vachon, C., Stevens, K.N., Lambrechts, D., Moisse, M., Paridaens, R., Christiaens, M.R., Rudolph, A., Nickels, S., Flesch-Janys, D., Johnson, N., Aitken, Z., Aaltonen, K., Heikkinen, T., Broeks, A., Veer, L.J., van der Schoot, C.E., Guenel, P., Truong, T., Laurent-Puig, P., Menegaux, F., Marme, F., Schneeweiss, A., Sohn, C., Burwinkel, B., Zamora, M.P., Perez, J.I., Pita, G., Alonso, M.R., Cox, A., Brock, I.W., Cross, S.S., Reed, M.W., Sawyer, E.J., Tomlinson, I., Kerin, M.J., Miller, N., Henderson, B.E., Schumacher, F., Le Marchand, L., Andrulis, I.L., Knight, J.A., Glendon, G., Mulligan, A.M., Lindblom, A., Margolin, S., Hooning, M.J., Hollestelle, A., van den Ouweland, A.M., Jager, A., Bui, Q.M., Stone, J., Dite, G.S., Apicella, C., Tsimiklis, H., Giles, G.G., Severi, G., Baglietto, L., Fasching, P.A., Haeberle, L., Ekici, A.B., Beckmann, M.W., Brenner, H., Muller, H., Arndt, V., Stegmaier, C., Swerdlow, A., Ashworth, A., Orr, N., Jones, M., Figueroa, J., Lissowska, J., Brinton, L., Goldberg, M.S., Labreche, F., Dumont, M., Winqvist, R., Pylkas, K., Jukkola-Vuorinen, A., Grip, M., Brauch, H., Hamann, U., Bruning, T., Radice, P., Peterlongo, P., Manoukian, S., Bonanni, B., Devilee, P., Tollenaar, R.A., Seynaeve, C., van Asperen, C.J., Jakubowska, A., Lubinski, J., Jaworska, K., Durda, K., Mannermaa, A., Kataja, V., Kosma, V.M., Hartikainen, J.M., Bogdanova, N.V., Antonenkova, N.N., Dork, T., Kristensen, V.N., Anton-Culver, H., Slager, S., Toland, A.E., Edge, S., Fostira, F., Kang, D., Yoo, K.Y., Noh, D.Y., Matsuo, K., Ito, H., Iwata, H., Sueta, A., Wu, A.H., Tseng, C.C., Van Den Berg, D., Stram, D.O., Shu, X.O., Lu, W., Gao, Y.T., Cai, H., Teo, S.H., Yip, C.H., Phuah, S.Y., Cornes, B.K., Hartman, M., Miao, H., Lim, W.Y., Sng, J.H., Muir, K., Lophatananon, A., Stewart-Brown, S., Siriwanarangsan, P., Shen, C.Y., Hsiung, C.N., Wu, P.E., Ding, S.L., Sangrajrang, S., Gaborieau, V., Brennan, P., McKay, J., Blot, W.J., Signorello, L.B., Cai, Q., Zheng, W., Deming-Halverson, S., Shrubsole, M., Long, J., Simard, J., Garcia-Closas, M., Pharoah, P.D., Chenevix-Trench, G., Dunning, A.M., Benitez, J., and Easton, D.F. 2013. Large-scale genotyping identifies 41 new loci associated with breast cancer risk. NATURE GENETICS 45:353-361, 361e. 10.1038/ng.2563

Oltean, S., and Bates, D.O. 2014. Hallmarks of alternative splicing in cancer. ONCOGENE 33:5311-5318. 10.1038/onc.2013.533

Pan, Q., Shai, O., Lee, L.J., Frey, B.J., and Blencowe, B.J. 2008. Deep surveying of alternative splicing complexity in the human transcriptome by high-throughput sequencing. NATURE GENETICS 40:1413-1415. 10.1038/ng.259

Sauerbrei, W., Royston, P., and Binder, H. 2007. Selection of important variables and determination of functional 
383

384

385

386

387

388

389

390

391

392

393

394

395

396

397

398

399

400

401

402

403

404

405

406

407

408

409

410

411

412

413

414

415

416

417

418

419

420

421

422

form for continuous predictors in multivariable model building. STATISTICS IN MEDICINE 26:5512-5528. $10.1002 / \operatorname{sim} .3148$

Spaethling, J.M., Sanchez-Alavez, M., Lee, J., Xia, F.C., Dueck, H., Wang, W., Fisher, S.A., Sul, J.Y., Seale, P., Kim, J., Bartfai, T., and Eberwine, J. 2016. Single-cell transcriptomics and functional target validation of brown adipocytes show their complex roles in metabolic homeostasis. FASEB JOURNAL 30:81-92. 10.1096/fj.15-273797

Tang, Y., Geng, Y., Luo, J., Shen, W., Zhu, W., Meng, C., Li, M., Zhou, X., Zhang, S., and Cao, J. 2015. Downregulation of ubiquitin inhibits the proliferation and radioresistance of non-small cell lung cancer cells in vitro and in vivo. Sci Rep 5:9476. 10.1038/srep09476

Tian, Y., Ding, W., Wang, Y., Ji, T., Sun, S., Mo, Q., Chen, P., Fang, Y., Liu, J., Wang, B., Zhou, J., Ma, D., and Wu, P. 2013. Ubiquitin B in cervical cancer: critical for the maintenance of cancer stem-like cell characters. PLoS One 8:e84457. 10.1371/journal.pone.0084457

Tibshirani, R. 1997. The lasso method for variable selection in the Cox model. STATISTICS IN MEDICINE 16:385395. 10.1002/(sici)1097-0258(19970228)16:4\&lt;385::aid-sim380\&gt;3.0.co;2-3

Tremblay, M.P., Armero, V.E., Allaire, A., Boudreault, S., Martenon-Brodeur, C., Durand, M., Lapointe, E., Thibault, P., Tremblay-Letourneau, M., Perreault, J.P., Scott, M.S., and Bisaillon, M. 2016. Global profiling of alternative RNA splicing events provides insights into molecular differences between various types of hepatocellular carcinoma. $B M C$ GENOMICS 17:683. 10.1186/s12864-016-3029-z

Villanueva, A. 2019. Hepatocellular Carcinoma. N Engl J Med 380:1450-1462. 10.1056/NEJMra1713263

Wan, L., Yu, W., Shen, E., Sun, W., Liu, Y., Kong, J., Wu, Y., Han, F., Zhang, L., Yu, T., Zhou, Y., Xie, S., Xu, E., Zhang, H., and Lai, M. 2019. SRSF6-regulated alternative splicing that promotes tumour progression offers a therapy target for colorectal cancer. GUT 68:118-129. 10.1136/gutjnl-2017-314983

Wang, E.T., Sandberg, R., Luo, S., Khrebtukova, I., Zhang, L., Mayr, C., Kingsmore, S.F., Schroth, G.P., and Burge, C.B. 2008. Alternative isoform regulation in human tissue transcriptomes. NATURE 456:470-476. 10.1038 /nature07509

Wu, H.C., Yang, H.I., Wang, Q., Chen, C.J., and Santella, R.M. 2017. Plasma DNA methylation marker and hepatocellular carcinoma risk prediction model for the general population. CARCINOGENESIS 38:1021-1028. 10.1093/carcin/bgx078

Yau, W.Y., Shih, H.C., Tsai, M.H., Sheu, J.C., Chen, C.H., and Chow, L.P. 2013. Autoantibody recognition of an Nterminal epitope of hnRNP L marks the risk for developing HBV-related hepatocellular carcinoma. Journal of Proteomics 94:346-358. 10.1016/j.jprot.2013.10.003

Yuan, J.H., Liu, X.N., Wang, T.T., Pan, W., Tao, Q.F., Zhou, W.P., Wang, F., and Sun, S.H. 2017. The MBNL3 splicing factor promotes hepatocellular carcinoma by increasing PXN expression through the alternative splicing of IncRNA-PXN-AS1. NATURE CELL BIOLOGY 19:820-832. 10.1038/ncb3538

Zhao, Q.J., Zhang, J., Xu, L., and Liu, F.F. 2018. Identification of a five-long non-coding RNA signature to improve the prognosis prediction for patients with hepatocellular carcinoma. World $J$ Gastroenterol 24:3426-3439. 10.3748/wjg.v24.i30.3426

Zhu, G.Q., Zhou, Y.J., Qiu, L.X., Wang, B., Yang, Y., Liao, W.T., Luo, Y.H., Shi, Y.H., Zhou, J., Fan, J., and Dai, Z. 2019. Prognostic alternative mRNA splicing signature in hepatocellular carcinoma: a study based on large-scale sequencing data. CARCINOGENESIS. 10.1093/carcin/bgz073

Peer] reviewing PDF | (2019:09:40919:1:1:NEW 5 Nov 2019) 
Figure 1

Flow chart.

Flow chart of the study design.

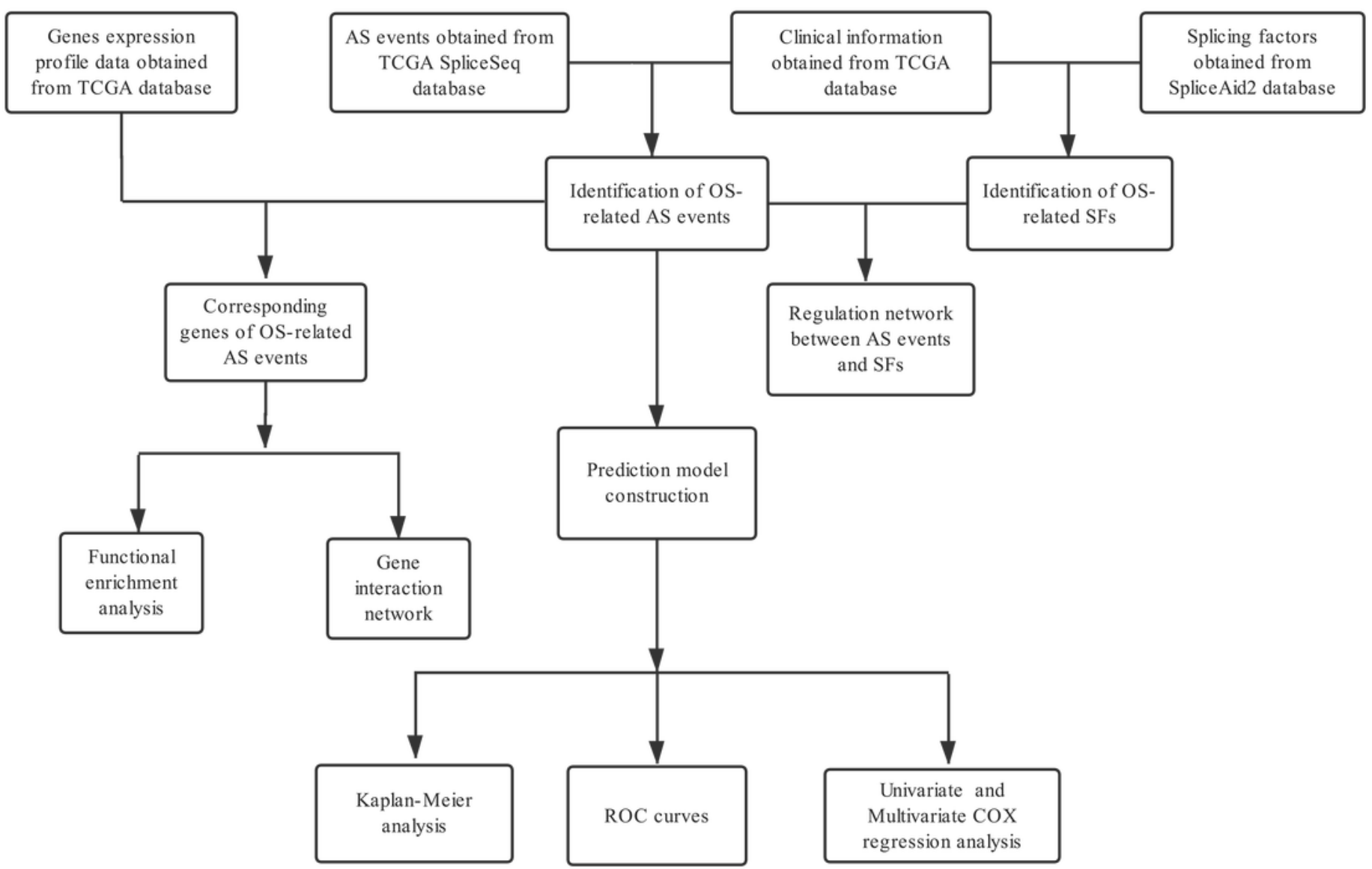


Figure 2

Overview of AS events in HCC patients.

(A) Upset plot of the intersections between the seven types of AS events. (B) Volcano plot of OS-related AS events (red dot) and OS-irrelated AS events (blue dot). (C) Upset plot of the intersections between the seven types of OS-related AS events.

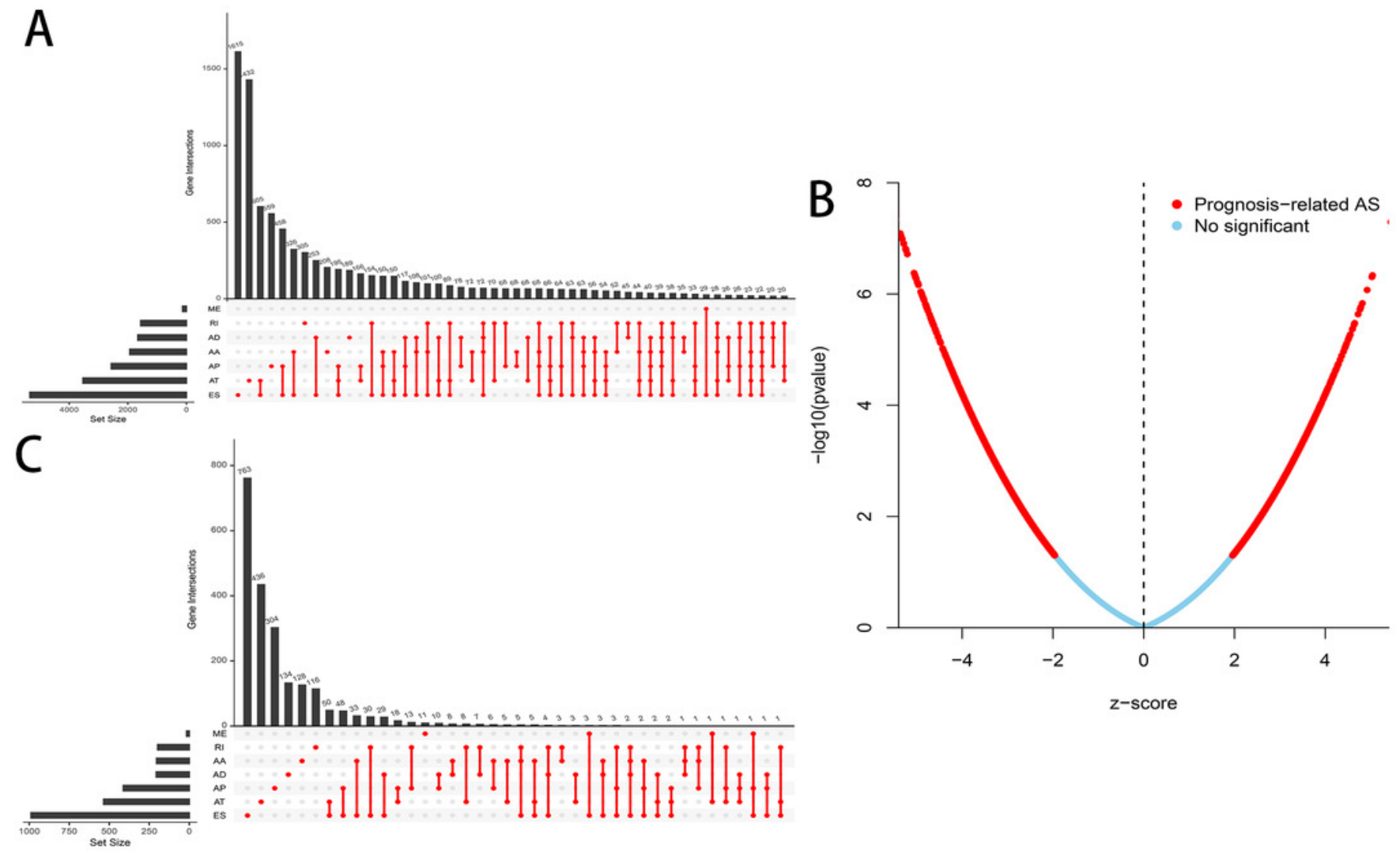


Figure 3

Bubble plots of OS-related AS events in HCC patients.

(A-F) The top 20 significant OS-related AS events for AA, AD, AP, AT, ES and RI. (G) 16 OSrelated AS events for ME.

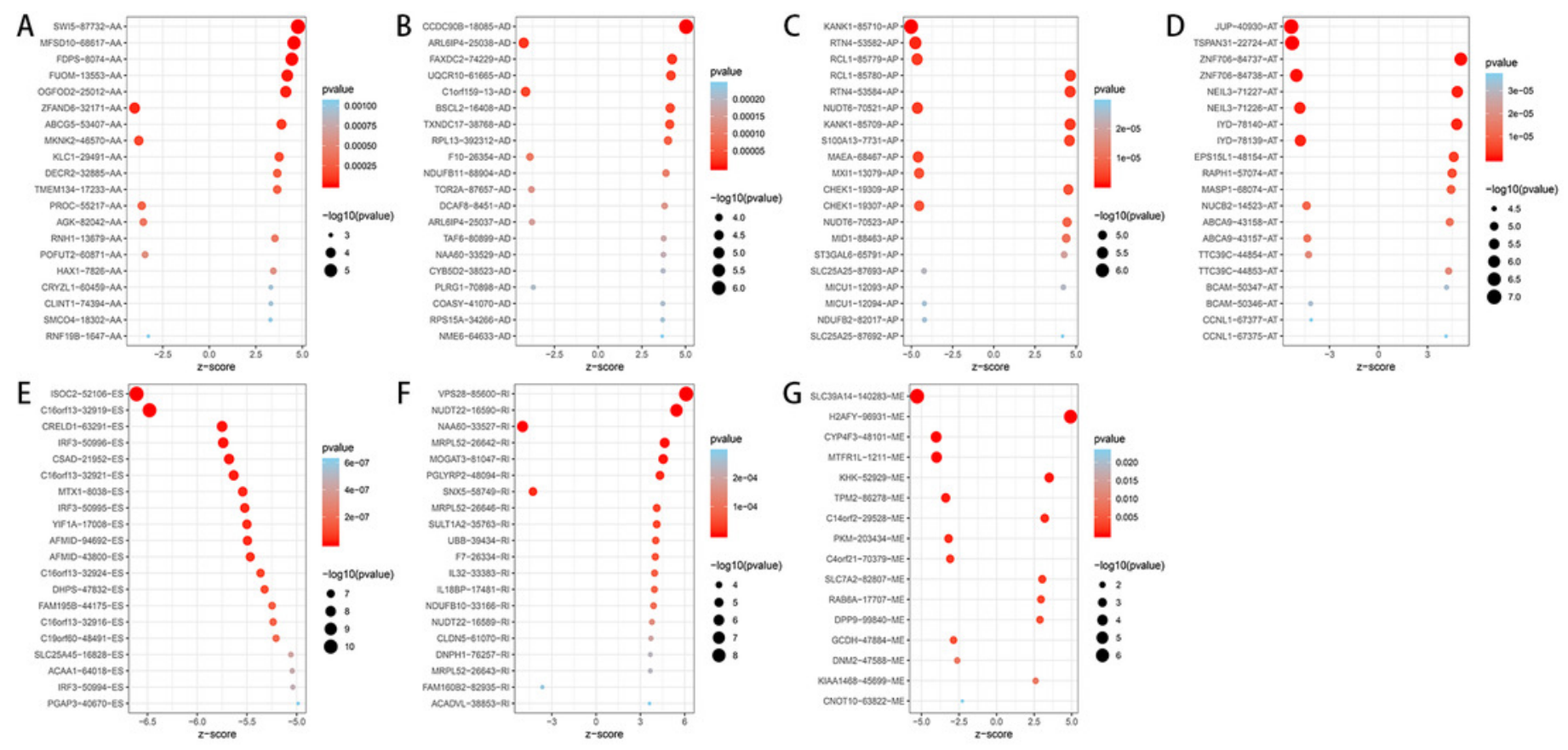




\section{Figure 4}

Gene interaction network and functional enrichment analysis.

(A) Gene interaction network of the top 800 most significant OS-related AS events. (B) The top 10 significant terms of GO enrichment analysis (BP/CC/MF). (C) Nine terms of KEGG enrichment analysis. 
A

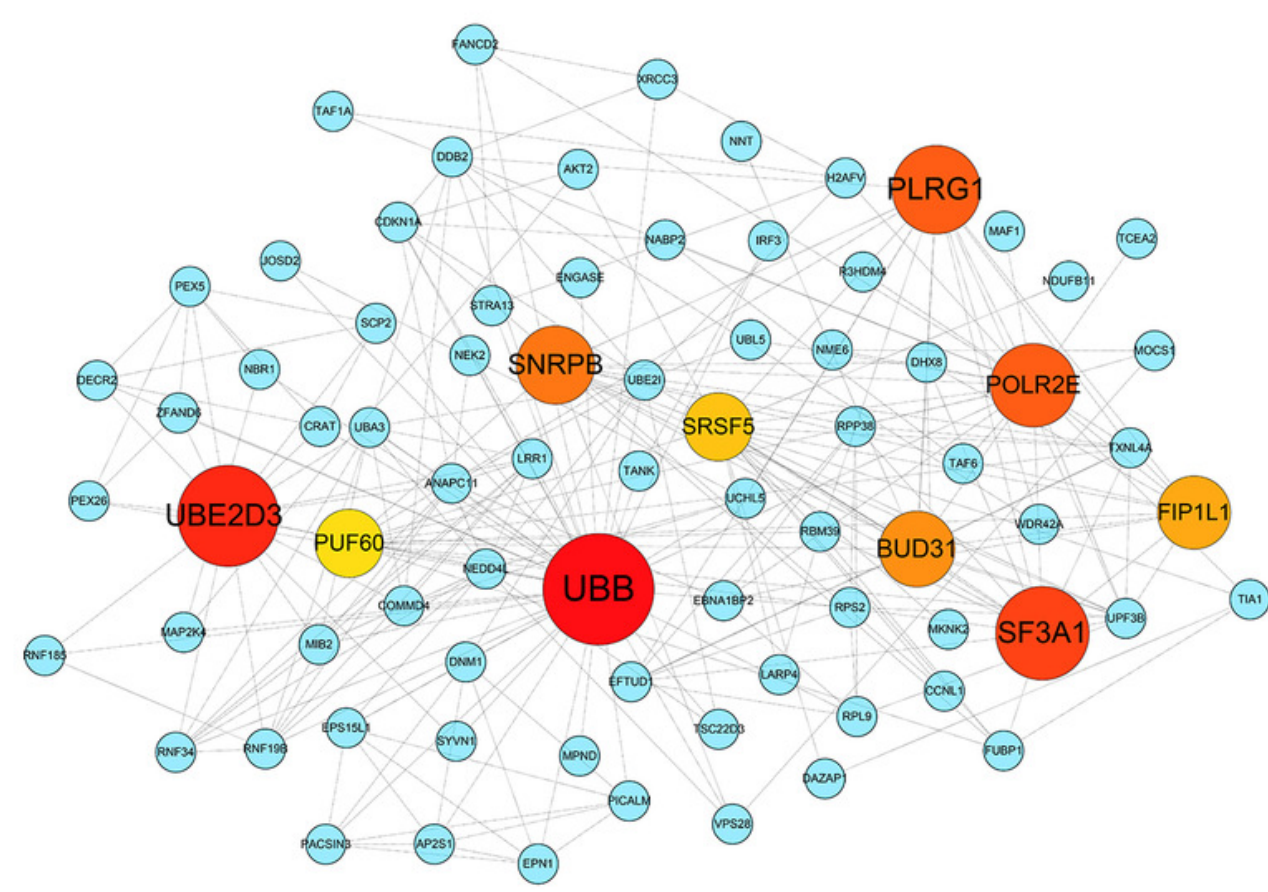

B
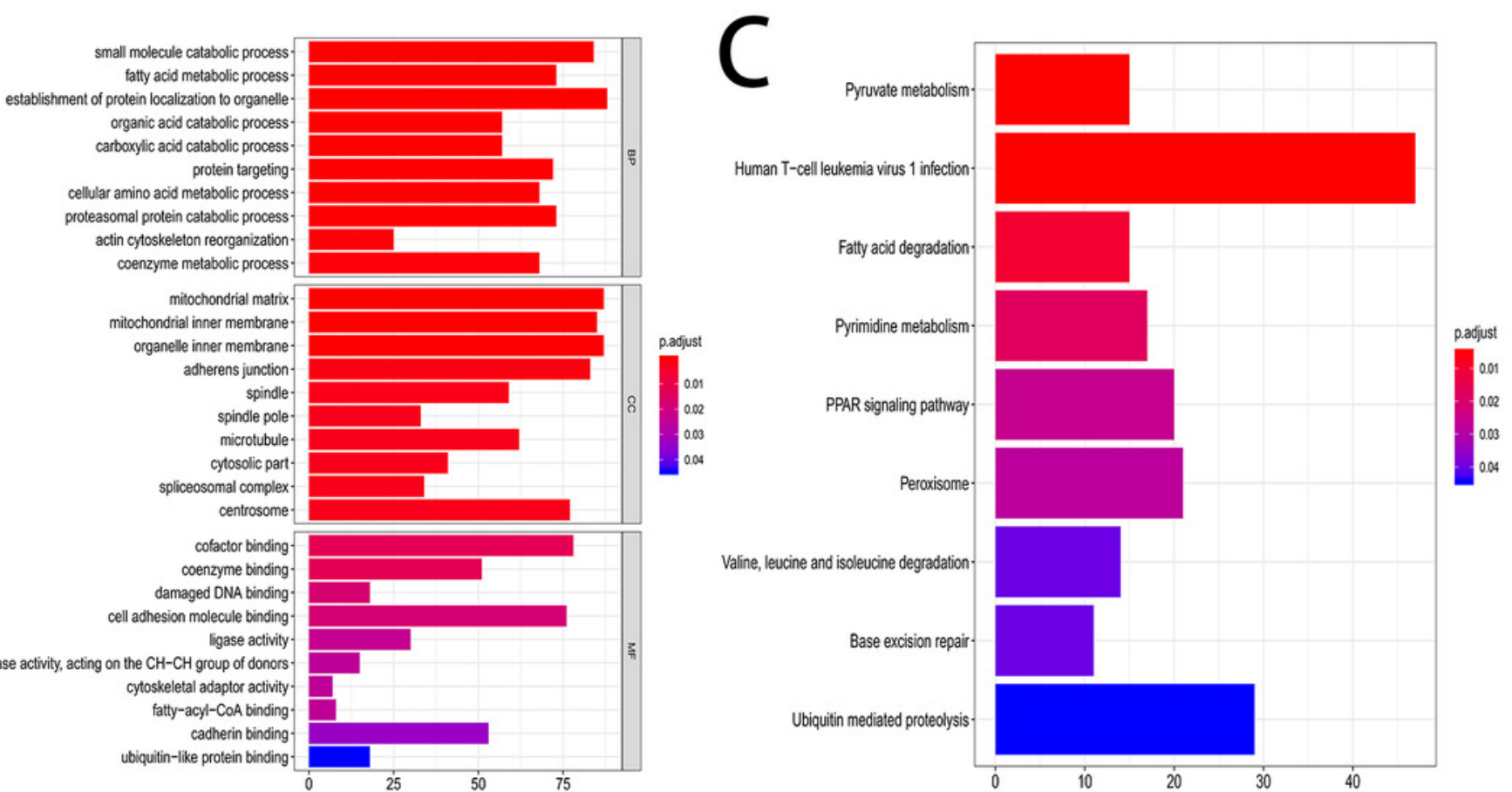
Figure 5

Selection of the optimal AS-related events used for construction of the final prediction model by LASSO regression

(A) Selection of optimal parameter (lambda) in the LASSO model, dotted vertical lines were drawn at the optimal values. (B) LASSO coefficient profiles of the nine OS-related AS events with nonzero coefficients determined by the optimal lambda.
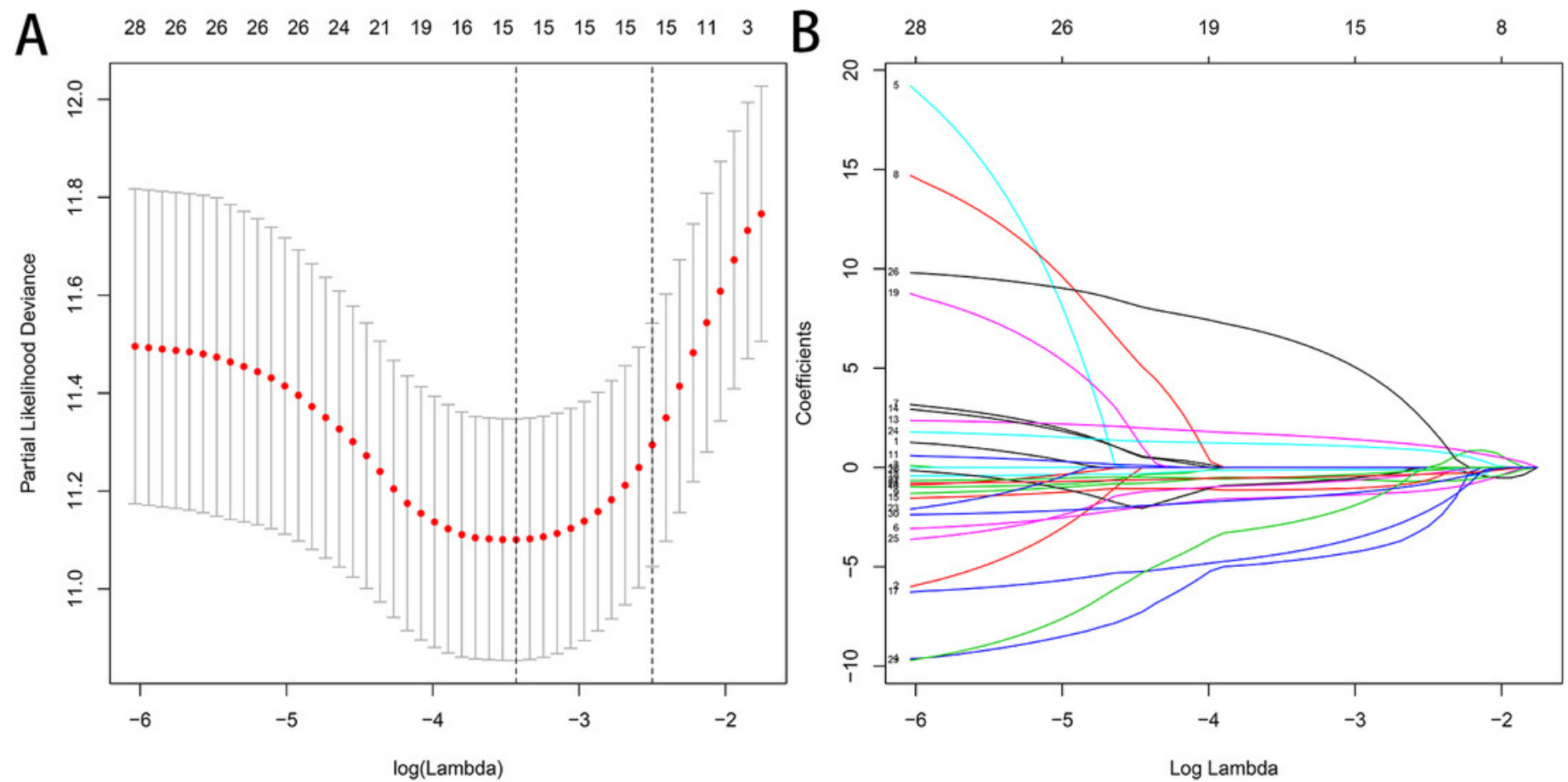


\section{Figure 6}

Analysis of the prediction models in HCC patients

HCC patients were divided into low and high risk groups on the basis of the median value of risk scores. (A-D, M-P) The risk score curves for $A A, A D, A P, A T, E S, R I, M E$ events and all types of AS events. (E-H, Q-T) Survival status and survival times of HCC patients ranked by risk score for $A A, A D, A P, A T, E S, R I, M E$ events and all types of $A S$ events. (I-L, U-X) The PSI value heatmap of the AS events for $A A, A D, A P, A T, E S, R I, M E$ events and all types of AS events. Color from blue to red indicates the increasing PSI score of corresponding AS event from low to high.

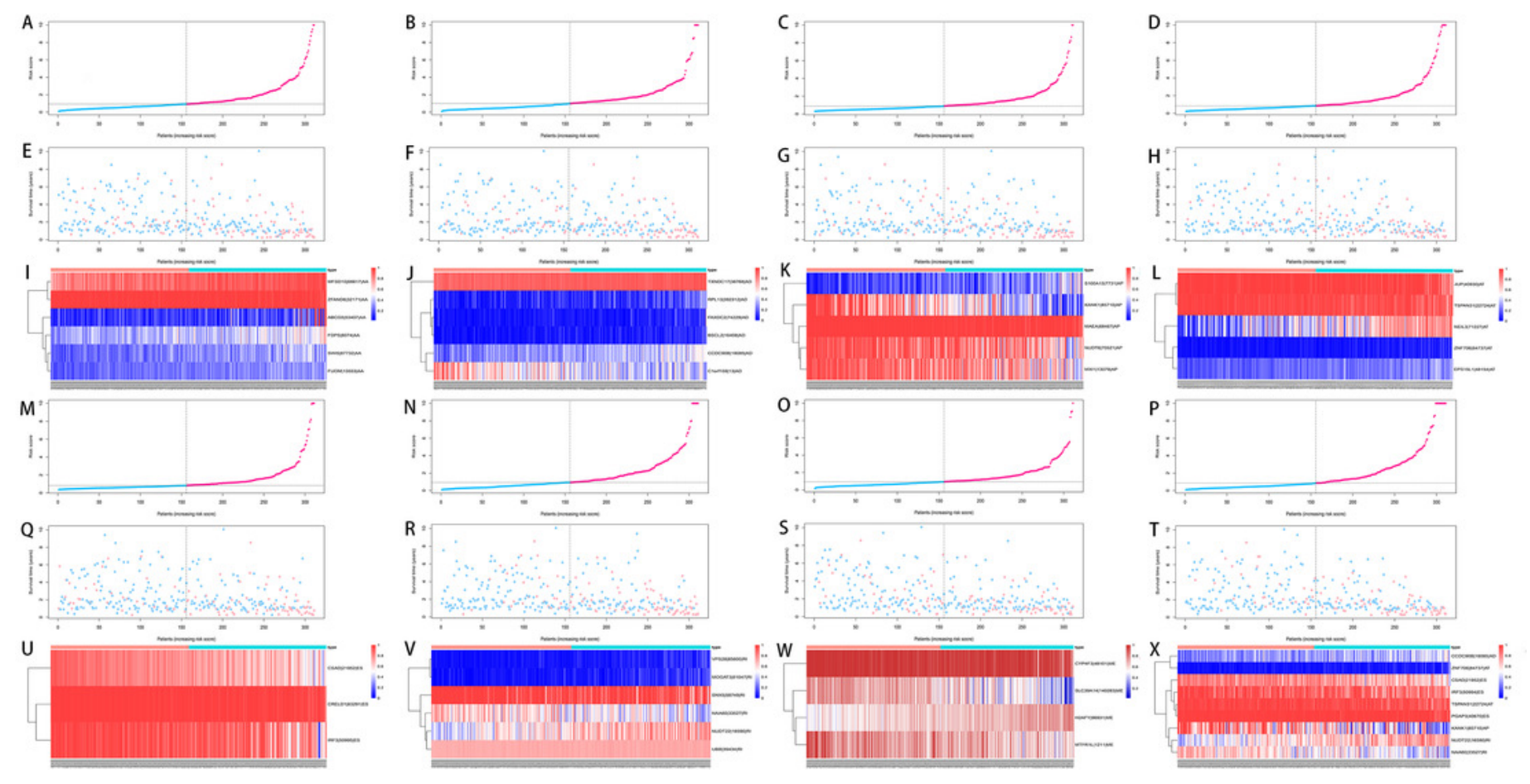


Figure 7

Kaplan-Meier plots of the eight prediction models constructed with AS events for HCC patients

(A-H) Kaplan-Meier plots of prediction models constructed with AA, AD, AP, AT, ES, RI, ME events and all types of AS events .
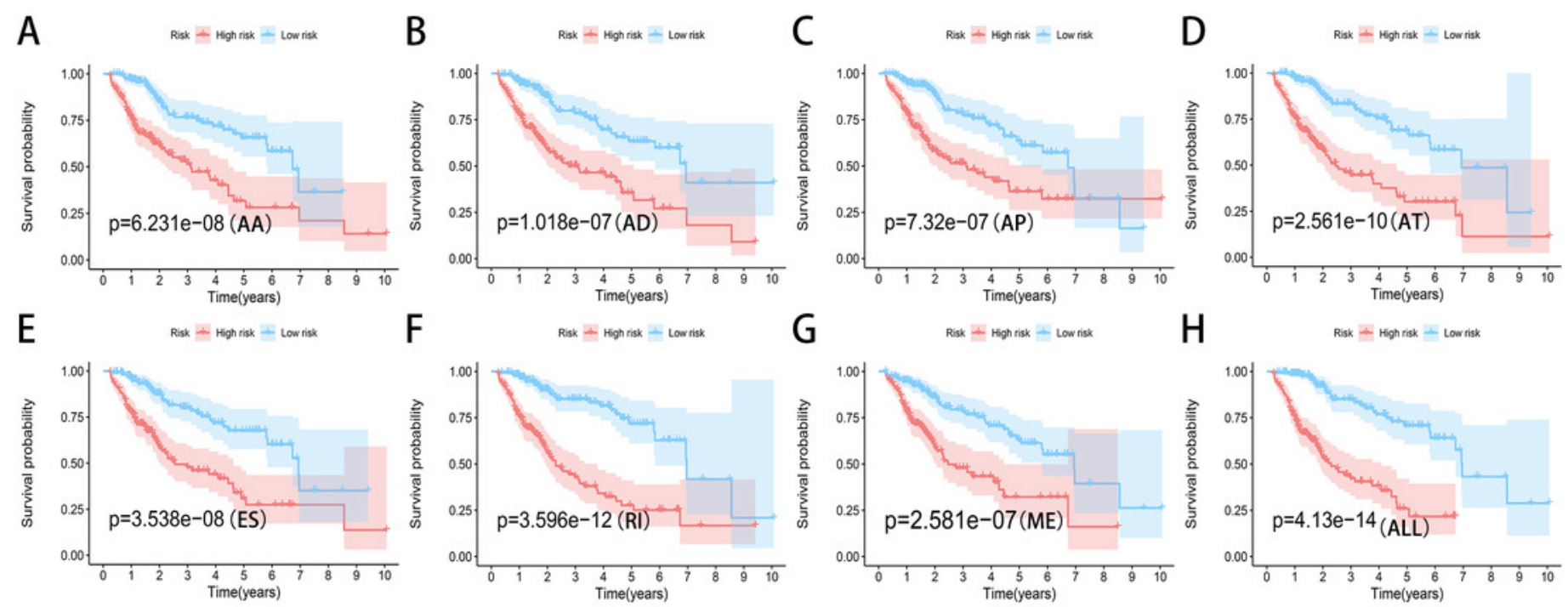
Figure 8

ROC curves of the eight prediction models for risk prediction in 1year, 3 years and 5 years.

ROC curves in 1 year (A-H), 3 years (I-P) and 5 years (Q-X) for AA, AD, AP, AT, ES, ME, RI models and the final model (All).
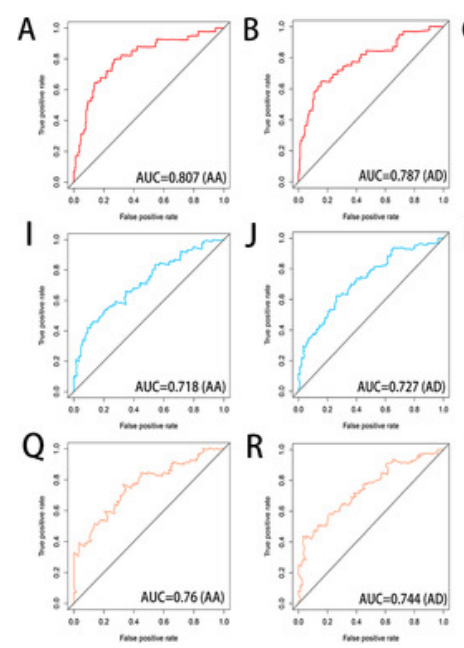
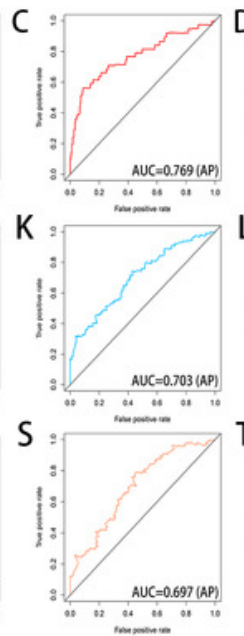
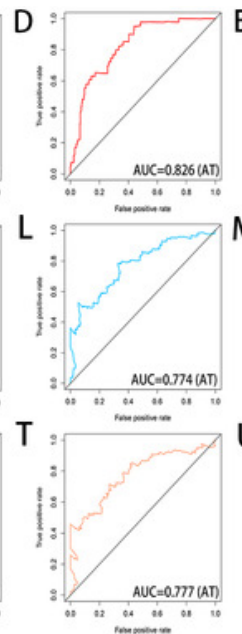
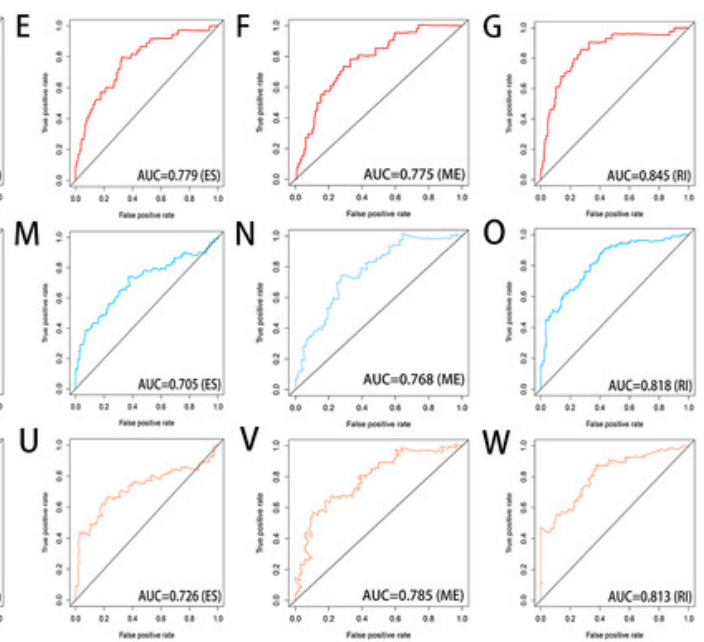
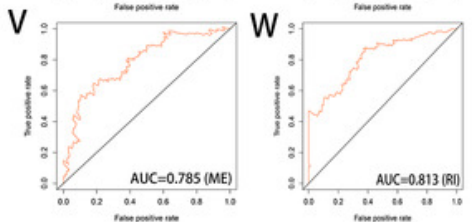
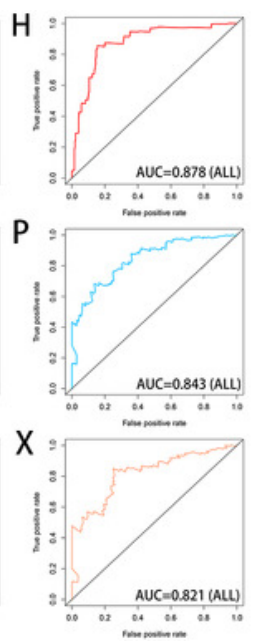


\section{Figure 9}

Forest plots of Cox regression analysis of clinicopathological characteristics and risk score (All).

(A) univariate analysis, (B) multivariate analysis.

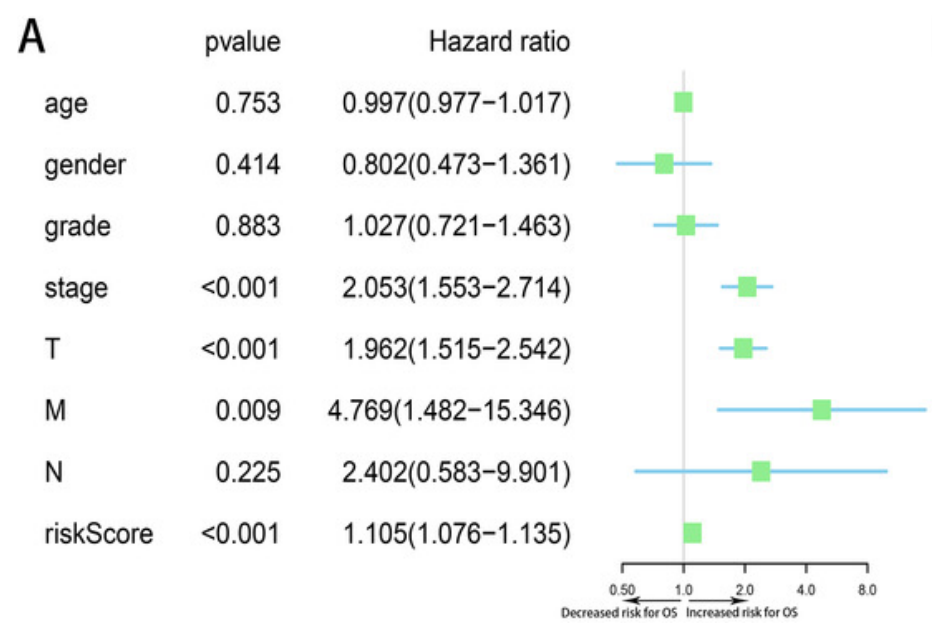

$\begin{array}{lrr}\text { B } & \text { pvalue } & \text { Hazard ratio } \\ \text { age } & 0.409 & 0.991(0.971-1.012) \\ \text { gender } & 0.623 & 0.859(0.467-1.577) \\ \text { grade } & 0.767 & 1.061(0.717-1.570) \\ \text { stage } & 0.894 & 0.926(0.297-2.887) \\ \mathrm{T} & 0.212 & 1.936(0.687-5.457) \\ \mathrm{M} & 0.500 & 1.600(0.408-6.271) \\ \mathrm{N} & 0.230 & 2.976(0.501-17.672) \\ \text { riskScore } & <0.001 & 1.101(1.069-1.135)\end{array}$

$1.101(1.069-1.135)$

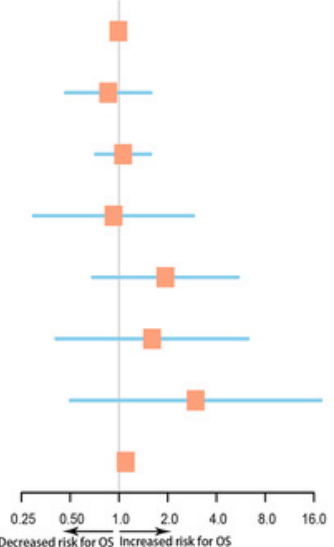




\section{Figure 10}

Regulation network of SFs and OS-related AS events.

(A) Correlation network between the expression of OS-related SFs and the PSI values of OSrelated AS events. The expression of OS-related SFs (blue dots) were positively (red line) or negatively (green line) correlated with the PSI values of OS-related AS events. The favorable AS events are showed by green dots while adverse AS events are showed by red dots. (B, F) Expression of HNRNPL and HNRNPH3 in tumor sample (red dots) and adjacent normal sample (blue dots). (C, G) Kaplan-Meier plots of HNRNPL and HNRNPH3 , HCC patients were divided into low (blue curve) and high risk (red curve) groups according to the median value of the two SFs expression. (D, E) Correlation analysis between the expression of HNRNPL and the PSI values of RTN4-53582-AP, RTN4-53584-AP . (H, I) Correlation analysis between the expression of HNRNPH3 and the PSI values of PCCB-66900-AT , PCCB-66901-AT . 

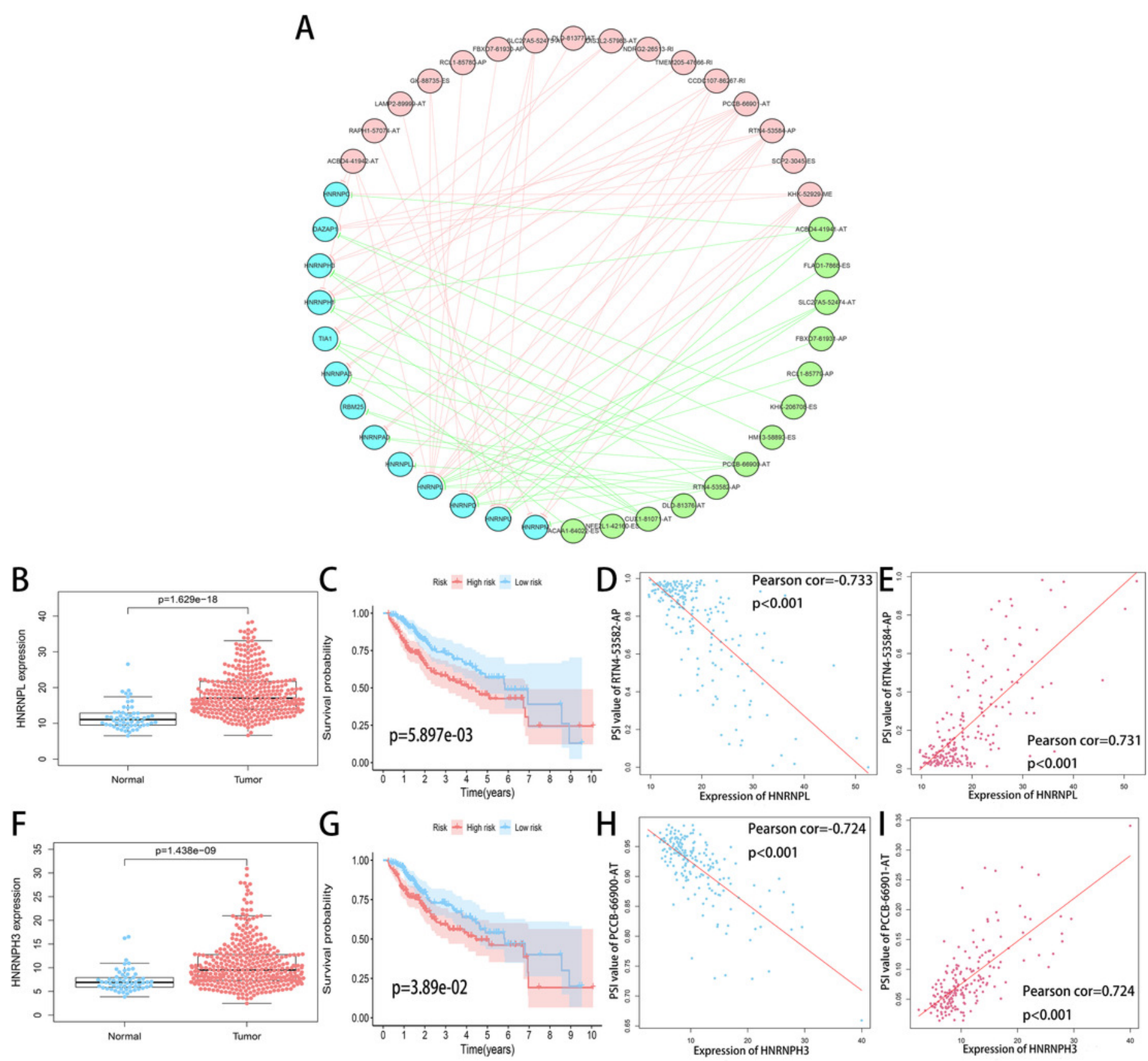


\section{Table 1 (on next page)}

Table 1

Overview of total AS events and OS-related AS events. 
1

2

3

4

Table 1 Overview of total AS events and OS-related AS events

\begin{tabular}{ccccc}
\hline \multirow{2}{*}{ Type } & \multicolumn{2}{c}{ Total AS events } & \multicolumn{2}{c}{ OS-related AS events } \\
\cline { 2 - 5 } & AS events & Genes & AS events & Genes \\
\hline AA & 2666 & 1937 & 217 & 203 \\
AD & 2331 & 1663 & 231 & 205 \\
AP & 6352 & 2566 & 643 & 411 \\
AT & 8087 & 3532 & 887 & 534 \\
ES & 12327 & 5343 & 1264 & 988 \\
ME & 137 & 135 & 16 & 16 \\
RI & 2263 & 1561 & 224 & 196 \\
ALL & 34163 & 8985 & 3482 & 2203 \\
\hline
\end{tabular}

5

6

7

8

9

10

11

12

13

14

15

16

17

18

19

20

21

22

23

24

25

26 
Table 2 (on next page)

Table 2

Information of AS events used for construction of prediction model. 
1 Table 2 Information of AS events used for construction of prediction model

\begin{tabular}{|c|c|c|c|c|c|c|}
\hline Type & ID & Coef & $\mathrm{HR}$ & $95 \%$ Lower & $95 \% \mathrm{Up}$ & $P$ value \\
\hline \multirow[t]{6}{*}{$\mathrm{AA}$} & SWI5-87732-AA & 3.44 & 31.34 & 4.32 & 227.57 & 0.00066043 \\
\hline & MFSD10-68617-AA & 5.53 & 251.81 & 4.79 & 13244.14 & 0.006246456 \\
\hline & FDPS-8074-AA & 1.72 & 5.58 & 0.96 & 32.52 & 0.055702088 \\
\hline & FUOM-13553-AA & 4.50 & 89.61 & 2.18 & 3689.99 & 0.017794651 \\
\hline & ZFAND6-32171-AA & -18.97 & $5.79 \mathrm{e}-9$ & $1.84 \mathrm{e}-14$ & $1.83 \mathrm{e}-3$ & 0.003324932 \\
\hline & ABCG5-53407-AA & 1.45 & 4.24 & 1.54 & 11.67 & 0.005133725 \\
\hline \multirow[t]{6}{*}{$\mathrm{AD}$} & CCDC90B-18085-AD & 2.73 & 15.27 & 2.46 & 94.69 & 0.003404273 \\
\hline & FAXDC2-74229-AD & 3.72 & 41.20 & 1.74 & 973.72 & 0.021200397 \\
\hline & C1orf159-13-AD & -2.02 & 0.13 & 0.04 & 0.42 & 0.000547079 \\
\hline & BSCL2-16408-AD & 6.33 & 560.69 & 9.32 & 33724.29 & 0.002462146 \\
\hline & TXNDC17-38768-AD & 12.15 & $1.89 \mathrm{e}+5$ & 8.72 & $4.12 \mathrm{e}+9$ & 0.017081663 \\
\hline & RPL13-392312-AD & 3.25 & 25.81 & 3.24 & 205.55 & 0.002134468 \\
\hline \multirow[t]{5}{*}{$\mathrm{AP}$} & KANK1-85710-AP & -1.12 & 0.33 & 0.16 & 0.68 & 0.003039732 \\
\hline & NUDT6-70521-AP & -1.40 & 0.25 & 0.09 & 0.71 & 0.009102069 \\
\hline & S100A13-7731-AP & 1.33 & 3.76 & 0.93 & 15.27 & 0.063560786 \\
\hline & MAEA-68467-AP & -11.36 & $1.16 \mathrm{e}-5$ & $1.41 \mathrm{e}-8$ & 0.01 & 0.000914974 \\
\hline & MXI1-13079-AP & -1.25 & 0.29 & 0.09 & 0.89 & 0.030826651 \\
\hline \multirow[t]{5}{*}{$\mathrm{AT}$} & JUP-40930-AT & -4.18 & 0.02 & $8.21 \mathrm{e}-4$ & 0.29 & 0.005110540 \\
\hline & TSPAN31-22724-AT & -5.98 & $2.52 \mathrm{e}-3$ & $9.11 \mathrm{e}-5$ & 0.07 & 0.000412724 \\
\hline & ZNF706-84737-AT & 9.10 & 8974.41 & 116.68 & 690281.40 & $3.99 \mathrm{e}-5$ \\
\hline & NEIL3-71227-AT & 1.45 & 4.26 & 1.78 & 10.19 & 0.001109076 \\
\hline & EPS15L1-48154-AT & 5.79 & 327.41 & 15.76 & 6803.41 & 0.000183173 \\
\hline \multirow[t]{3}{*}{ ES } & CRELD1-63291-ES & -13.64 & $1.19 \mathrm{e}-6$ & $5.85 \mathrm{e}-10$ & $2.44 \mathrm{e}-3$ & 0.000452970 \\
\hline & CSAD-21952-ES & -3.21 & 0.04 & 0.01 & 0.19 & $4.24 \mathrm{e}-5$ \\
\hline & IRF3-50995-ES & -2.62 & 0.07 & 0.03 & 0.20 & $6.14 \mathrm{e}-7$ \\
\hline \multirow[t]{4}{*}{$\mathrm{ME}$} & SLC39A14-140283-ME & -1.96 & 0.14 & 0.04 & 0.45 & 0.001037644 \\
\hline & H2AFY-96931-ME & 3.18 & 24.02 & 2.71 & 211.85 & 0.004205834 \\
\hline & CYP4F3-48101-ME & -4.59 & 0.01 & $2.74 \mathrm{e}-4$ & 0.37 & 0.012551164 \\
\hline & MTFR1L-1211-ME & -2.30 & 0.10 & 0.03 & 0.38 & 0.000662517 \\
\hline \multirow[t]{5}{*}{ RI } & VPS28-85600-RI & 6.06 & 429.08 & 5.62 & 32758.26 & 0.006135308 \\
\hline & NUDT22-16590-RI & 2.69 & 14.79 & 3.18 & 68.86 & 0.000599344 \\
\hline & NAA60-33527-RI & -1.87 & 0.15 & 0.04 & 0.65 & 0.010841633 \\
\hline & MOGAT3-81047-RI & 5.24 & 188.66 & 5.22 & 6812.78 & 0.004190482 \\
\hline & SNX5-58749-RI & -3.94 & 0.02 & $3.24 \mathrm{e}-3$ & 0.12 & $1.70 \mathrm{e}-5$ \\
\hline
\end{tabular}




\begin{tabular}{ccccccc}
\hline \multirow{3}{*}{ ALL } & UBB-39434-RI & 44.68 & $2.54 \mathrm{e}+19$ & $2.40 \mathrm{e}+10$ & $2.69 \mathrm{e}+28$ & $2.50 \mathrm{e}-5$ \\
& CSAD-21952-ES & -2.15 & 0.12 & 0.02 & 0.58 & 0.008527832 \\
NUDT22-16590-RI & 2.26 & 9.58 & 2.24 & 40.90 & 0.00228534 \\
TSPAN31-22724-AT & -6.17 & $2.08 \mathrm{e}-3$ & $4.74 \mathrm{e}-5$ & 0.09 & 0.001376182 \\
CCDC90B-18085-AD & 1.53 & 4.62 & 0.68 & 31.15 & 0.116422196 \\
IRF3-50994-ES & -1.71 & 0.18 & 0.03 & 1.02 & 0.053074975 \\
ZNF706-84737-AT & 8.94 & 7608.05 & 76.67 & 754961.11 & 0.000138991 \\
KANK1-85710-AP & -0.80 & 0.45 & 0.22 & 0.94 & 0.033403 \\
PGAP3-40670-ES & -6.81 & $1.11 \mathrm{e}-3$ & $4.06 \mathrm{e}-7$ & 3.02 & 0.091751791 \\
NAA60-33527-RI & -2.27 & 0.10 & 0.03 & 0.41 & 0.001319454 \\
\hline
\end{tabular}

2

3 